\title{
Centralized Resource Allocation with MOLP Structure
}

\author{
R. Shamsi ${ }^{1 *}$, G. R. Jahanshahloo', M. R. Mozaffari ${ }^{2}$ and F. Hosseinzadeh Lotfi' \\ 'Department of Mathematics, Science and Research Branch, Islamic Azad University, Tehran, Iran; \\ shamsi_1195@yahoo.com,jahanshahloo@yahoo.com, farhad@hosseinzadeh.com \\ 2Department of Mathematics, Science and Research Branch, Islamic Azad University, Fars, Iran; \\ mozaffari854@yahoo.com
}

\begin{abstract}
In Data Envelopment Analysis (DEA), in addition to the efficiency score, the projection of inefficient Decision Making Units (DMUs) is also determined. In this paper, a model is proposed by using Centralized Resource Allocation (CRA) and Multi Objective Linear Programming (MOLP). In order to solve the proposed Multi objective model, we use the entropy and Z-W methods and obtain the projection of inefficient DMUs. The advantage of the proposed model, in addition to employing interactive methods, is that by solving one model instead of $n$ models; the projection is obtained for all DMUs, which is closer to reality and more practical. Finally, a numerical example and an application are provided and the interactive and entropy methods are utilized.
\end{abstract}

Keywords: DEA, Entropy, MOLP, Z-W

\section{Introduction}

Evaluating the efficiency of the production units of a system is an important issue for managers. Charnes et al. ${ }^{6}$ introduced Data Envelopment Analysis (DEA) to measure the relative efficiency of a set Decision Making Units (DMUs).

Lozano and Villa ${ }^{18}$ presented two centralized resource allocation BCC (CRA-BCC) models in a decision-making environment. Centralized resource allocation is situation in which all the DMUs fall under the umbrella of a centralized decision maker that oversees them. This type of situation occurs whenever all of the units belong to the same organization (public or private), which provides the units with the necessary resources to obtain their outputs. Many Data Envelopment Analysis (DEA) applications (such as those by bank branches, hospitals, university departments, supermarket chains and police stations) fall into this category. Asmiled et al. reconsidered one of the centralized models proposed by Lozano and villa ${ }^{17,19}$. Mar-Molinero et al. ${ }^{22}$ developed simplified version of the CRA-BCC model by Lozano and villa ${ }^{17}$. Other extension to the basic centralized resource allocation model includes that of Hosseinzadeh lotfi et al. ${ }^{11}$, Yu et al. ${ }^{3}$ and Lei Fang ${ }^{8}$.

In recent years, the relation between data envelopment analysis (DEA) and Multiple Objective Linear Programming (MOLP) has been of considerable importance to researchers. The structures of these two types of models have much in common, but DEA is directed to assessing past performances as part of the management control function and MOLP to planning future performances ${ }^{5}$. There exist some studies about the similarities between DEA and Multiple Criteria Decision Analysis (MCDA), generally, and MOLP, in particular. Doyle and Green $^{7}$ ( indicated that DEA is an MCDA method itself. Various models have been established to actively involve DMs in the target setting process in DEA, including Golany's', Thanassoulis and Dyson's ${ }^{12}$ and Athanassopoulos's ${ }^{1}$ models. Golany ${ }^{9}$ first established an interactive model involving both DEA and MOLP approaches. Yang et al. ${ }^{4}$ proposed three equivalence models in MOLP, including the super-ideal point model, the ideal point model, and the shortest distance model. Hosseinzadeh lotfi et al. ${ }^{11}$ studied relationship between MOLP and DEA.

*Author for correspondence 
Lotfi et al. ${ }^{11,13}$, Yang et al. ${ }^{4}$, Lozano and Villa ${ }^{19}$, Jablonsky ${ }^{16}$, Hadad et al. ${ }^{10}$ carried out research on obtaining the target using MOLP. Nowadays, managers in every organization try to make optimal use of the resources and capacities available to them. Performance evaluation of DMUs and finding suitable targets that are consistent with the surrounding environment and practicable, as well, is critical. Using multi objective models and interactive methods is undoubtedly essential to achieving the goals of an organization. Therefore, an MOLP is proposed by whose solution through the interactive and entropy methods a preferred for all DMUs is obtained.

This paper proceeds as follows: we present a brief introduction of DEA, MOLP, and CRA in section 2. We provide our proposed model in section 3 and examine its properties and related theorems. In section 4 , we state two methods for solving the proposed model: the Z-W method and the entropy method. Then, we provide a numerical example and an application. Finally, section 6 contains conclusions and some suggestions for future research.

\section{Preliminare}

In this section, a brief description of Data Envelopment Analysis and centralized resource allocation multiple objective linear programming is provided. A close study of the Pareto optimal solution of MOLP and the efficient units of DEA can be useful in understanding the relation between MOLP and DEA, considering their similar structures.

\subsection{Overview of DEA and CRA}

Consider $n$ DMUs with $m$ inputs and $s$ outputs. The input and output vectors of $D M U_{j}(j=1, \ldots, n)$ are $X_{j}=\left(x_{1 j}, \mathrm{~K}, x_{m j}\right), Y_{j}=\left(y_{1 j}, \mathrm{~K}, y_{s j}\right)$, respectively, where $X_{j}{ }^{3} 0, X_{j}{ }^{1} 0, Y_{j}{ }^{3} 0, Y_{j}{ }^{1} 0$. We define the most general production possibility set $\mathrm{T}$ as follows:

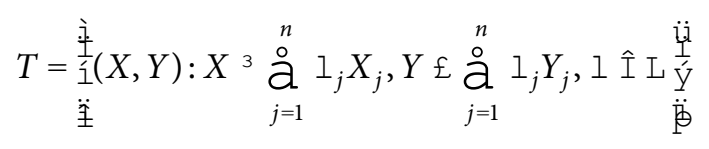

where $\Lambda$ is one of the following:

$$
\begin{aligned}
& \mathrm{L}_{C}=\left\{I \mid I^{3} 0\right\} \\
& \mathrm{L}_{v}=\left\{1 \mid 1 I=1, I^{3} 0\right\} \\
& \mathrm{L}_{N 1}=\left\{I \mid 11 £ 1, I^{3} 0\right\} \\
& \mathrm{L}_{N D}=\left\{I \mid 11{ }^{3} 1, I^{3} 0\right\} \text { where } I=\left(I_{1}, K, I_{n}\right) \hat{I} R^{n} .
\end{aligned}
$$

Therefore, we obtain four production possibility sets, in which we denote $T$ by $T_{c}, T_{v}, T_{N I}, T_{N D}$, when I $\hat{\mathrm{I}} T_{c}$, I $\hat{\mathrm{I}} T_{v}, \perp \hat{\mathrm{I}} T_{N I}, \perp \hat{\mathrm{I}} T_{N D}$, respectively. When a $D M U_{o}, o \hat{\mathrm{I}}\{1,2, \mathrm{~K}, n\}$, is under evaluation, we use the input-oriented DEA model proposed by Banker as follows:

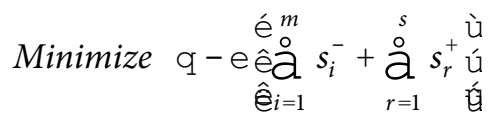

Subject to

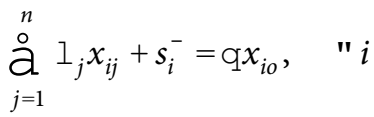

$$
\begin{aligned}
& \stackrel{\bigotimes}{j=1}_{j}^{n} l_{j} y_{r j}-s_{r}^{+}=y_{r o}, \quad " r
\end{aligned}
$$

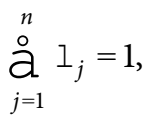

$$
\begin{aligned}
& l_{j}{ }^{3} 0, \quad " j \\
& s_{i}^{-3} 0, s_{r}^{+3} 0, \quad " i, r \text {. }
\end{aligned}
$$

where $>0$, is a so-called non-Archimedean element defined to be smaller than any positive real number. This is equivalent to solving (1) in two stages by first minimizing , then fixing $={ }^{*}$ where the slacks are to be maximized without altering the previously determined value of $={ }^{*}$. Clearly, the evaluated $D M U_{O}$ is efficient if $=1^{*}$ and only if and all slack variables in the optimal solution are zero in problem (1).

The Centralized Resource Allocation BCC model (CRA-BCC) has been introduced by Lozano and Villa ${ }^{18}$ that by solving one model instead of $\mathrm{n}$ models, the projection is obtained for all DMUs. (CRA-BCC) is a Data Envelopment Analysis (DEA)-type model as follows:

$$
\begin{aligned}
& \text { Minimize } \\
& \text { Subject to }
\end{aligned}
$$

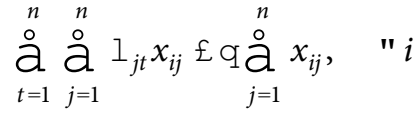

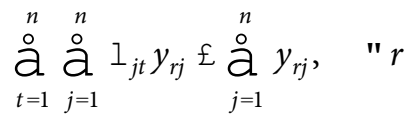

$$
\begin{aligned}
& \stackrel{\circ}{a}_{j=1}^{n} 1_{j t}=1, \quad " t \\
& 1_{j t}{ }^{3} 0 \text {, q free, } \quad " j, t
\end{aligned}
$$


Once the model is solved, the corresponding vector $\left(I_{1 t}^{*}, \mathrm{~K}, I_{n t}^{*}\right)$ defines for each $D M U_{t}$ the operating point at which it should aim. The inputs and outputs of each such point can be computed as

$$
\begin{aligned}
& \bar{x}_{i t}=\stackrel{Ð}{j=1}_{j}^{n} 1_{j t}^{*} x_{i j}, \quad " i
\end{aligned}
$$

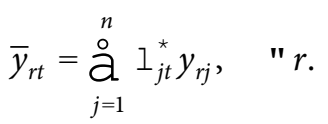

That For any $D M U_{t}$, the operating point onto which it is projected by Model CCR/Radial/Input-oriented $\left(\bar{x}_{1 t}, \bar{x}_{2 t}, \mathrm{~K}, \bar{x}_{m t}, \bar{y}_{1 t}, \bar{y}_{2 t}, \mathrm{~K}, \bar{y}_{s t}\right)$ is Pareto Efficient ${ }^{1}$.

\subsection{Overview of MOLP}

A Multi-Objective Linear Programming (MOLP) problem is to optimize a vector of linear functions in the presence of linear constraints and can be formulated as follows:

$$
\begin{aligned}
& \text { Maximize }\left(f_{1}(x), f_{2}(x), \ldots, f_{1}(x)\right) \\
& \text { Subject to } \\
& \text { Ax }=b \\
& x \geq 0
\end{aligned}
$$

where $f_{i}(x)=c^{i} x, i=1,2, \ldots, l$ are the objective functions, $A \in R^{m \times n}$ is the constraint matrix, $b \in R^{m}$ is the right-hand side vector and $x \in R^{n}$ is the vector of variables. We shall denote the feasible set of the (1) by X. In the following we assume, without loss of generality, that $\mathrm{X}$ is non empty. The objective function can be written as $C^{T} x$, where $C \in R^{m x i}$ has columns $c^{i}$. A solution $x^{*} \in X$ of (1) is (weakly) Pareto optimal if there is no $x \in X$ such that $C^{T} x \geq C^{T} x^{*}$ and $C^{T} x \neq C^{T} x^{*}$. Let

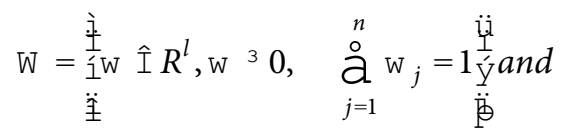

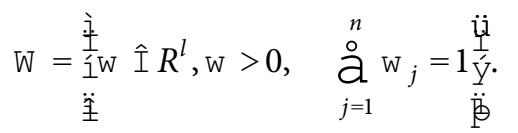

\section{THEOREM 1}

A feasible point $x \in X$ is (weakly) Pareto optimal for (3) if and only if there exists $\in \mid(\lambda \in \mid)$ such that $\mathrm{x}$ is an optimal solution of

$$
\begin{aligned}
& \text { Maximize } \stackrel{\circ}{a=1}^{n} \mathrm{w}_{i}\left(c^{i}\right)^{T}(x) \\
& \text { Subject to } \\
& \begin{array}{l}
\text { Ax=b, } \\
x \geq 0
\end{array}
\end{aligned}
$$

The proof can be found in Steuer ${ }^{20}$.

For more details about multi-objective optimization, readers are referred to Steuer ${ }^{12}$ and Hwang and Masud ${ }^{15}$.

\section{CRA Model with MOLP Structure}

Considering that decision maker preferences are applied in MOLP methods and the preferred point is obtained, which is more realistic and practical, the multi objective CRA model is proposed as follows:

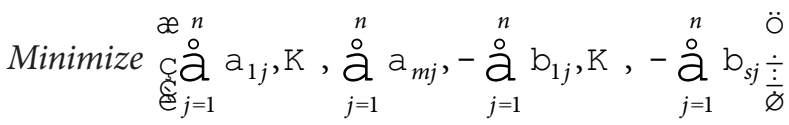

Subject to

$$
\begin{aligned}
& \stackrel{n}{\stackrel{n}{a}} \stackrel{n}{\stackrel{\circ}{a}} 1_{r j} x_{i j} £ \stackrel{\circ}{\stackrel{\circ}{a}} \mathrm{a}_{i j}, \quad \text { " } i \\
& \stackrel{\circ}{a}_{r=1}^{n} \stackrel{\circ}{j}_{j=1}^{n} 1_{r j} y_{k j}{ }^{3} \stackrel{\circ}{a}_{j=1}^{n} b_{k j}, \quad " k \\
& \stackrel{n}{\stackrel{n}{a}} 1_{r j}=1, \quad " j \\
& l_{r j}{ }^{3} 0, \quad " r, j \text {. }
\end{aligned}
$$

where $\left(l_{r j}, \mathrm{a}_{i j}, \mathrm{~b}_{r j}\right)$ are variables. We call this proposed model the MOLP-CRA model.

\section{THEOREM 2}

The model (5) has a feasible solution.

\section{ProOF:}

$z_{0}=\left(1_{r j}=1 " r=j, 1_{r j}=0 " r^{1} j, a_{i j}=x_{i j} " i, \mathrm{~b}_{k j}=y_{k j} " k\right)$ is the feasible solution of the model (5) because we have

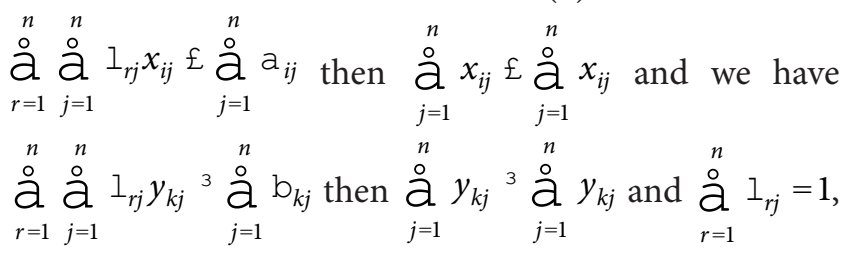
so $\mathrm{z}_{0}$ satisfies the constraints of model (6)

\section{THEOREM 3}

Each Pareto optimal solution of model (5) is corresponding to an efficient production possibility in $\mathrm{T}_{v}$. 


\section{Proof:}

Suppose $\left(I_{r j}^{*}, a_{i}^{*}, b_{k}^{*}\right)$ for i, j, r, k is a Pareto optimal solution of model (5) where $\mathrm{a}_{i}=\stackrel{n}{\mathrm{a}} \mathrm{a}_{i j}, \mathrm{~b}_{k}=\stackrel{n}{\mathrm{a}} \mathrm{b}_{k j}$. We want to prove $\left(\alpha^{*},{ }^{*}\right)$ is an efficient unit in $\mathrm{T}_{v}^{j=1}$. By contradiction, suppose $\left(\alpha^{*},{ }^{*}\right)$ is not an efficient unit in $T_{v}$. So, there exists $(\bar{X}, \bar{Y}) \hat{\mathrm{I}} T_{v}$ and $(-\bar{X}, \bar{Y})^{3}\left(-\mathrm{a}^{*}, \mathrm{~b}^{\star}\right)$, $(-\bar{X}, \bar{Y})^{1}\left(-\mathrm{a}^{*}, \mathrm{~b}^{*}\right)$. Therefore $(\bar{X}, \bar{Y}) \hat{\mathrm{I}} T_{v}$ so $\bar{I}$ exists such that $\bar{x}_{i}=\stackrel{\bigcirc}{a}_{j=1}^{n} \bar{l}_{i} x_{i j}, \bar{y}_{k}=\stackrel{\bigcirc}{a}_{j=1}^{n} \bar{l}_{j} y_{k j}$ and $\left(I_{r j}=\bar{I}_{j} " r, j, \bar{X}, \bar{Y}\right) i$ is a feasible solution model (5) which is a contradiction.

\section{THEOREM 4}

In each Pareto optimal solution of model (5) all input and output constraints are binding.

\section{Proof:}

Suppose $\left(I_{r j}^{*}, a_{i}^{*}, b_{k}^{*}\right)$ for i, j, r, k is a Pareto optimal

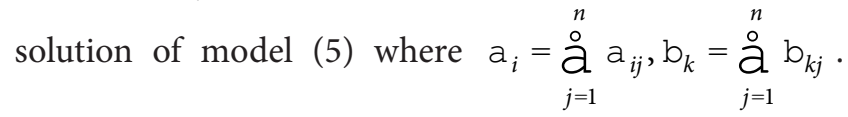
By contradiction, without loss of generality, assume that there exists $t \in\{1,2, \ldots, m\}$ such that $\stackrel{D}{a}_{r=1}^{n} \stackrel{D}{a}=1_{j=1}^{n} 1_{r j}^{*} x_{t j}<a_{t}^{*}$.

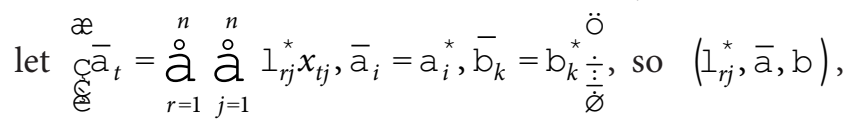
is a feasible solution for model (5) such that $(-\bar{a}, \bar{b})^{3}\left(-a^{*}, b^{*}\right),(-\bar{a}, \bar{b})^{1}\left(-a^{*}, b^{*}\right)$ which is a contradiction and completes the proof.

\section{THEOREM 5}

Each DMU is corresponding to an extreme point model (5).

\section{Proof:}

Suppose $D M U_{i}=\left(x_{l}, y_{l}\right)$. Let $Z=\left(I_{r j}=1, a_{i j}=1, b_{k j}=1, " j=l\right.$, $\left.I_{r j}=0, a_{i j}=1, b_{i j}=0 " j^{1} l\right)$, feasible solution for model (5). We want to prove $\mathrm{Z}$ is an extreme point. Because the basic matrix corresponding to the feasible solution $\mathrm{Z}$ is as follows:

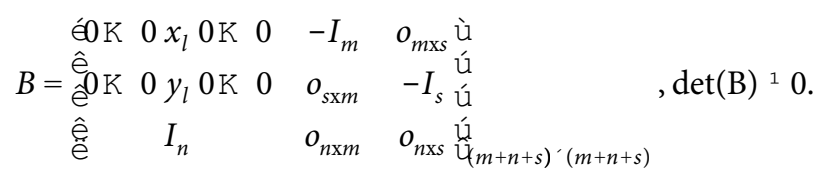

So $B$ is a basic feasible solution of model (5).

One of the methods to solve an MOLP problem is the weighted sum method. The weighted form of model (5) is as follows:

$$
\text { Minimize } \stackrel{\circ}{a}_{i=1}^{m} \stackrel{\circ}{a}_{j=1}^{n} w_{i} \mathrm{a}_{i j}-\stackrel{\circ}{\stackrel{\circ}{a}} \stackrel{\circ}{a}_{j=1}^{n} w_{k=1}^{\mathrm{S}_{k j}}
$$

Subject to

$$
\begin{aligned}
& -\stackrel{\circ}{a} \stackrel{\circ}{a}_{r=1}^{n} 1_{r j} x_{i j}+\stackrel{\stackrel{\circ}{a}}{a} a_{i j}{ }^{3} 0, \quad \text { " } i \\
& \stackrel{n}{\stackrel{\circ}{a}} \stackrel{\circ}{\stackrel{\circ}{a}} 1_{r j} y_{k j}-\stackrel{\circ}{\stackrel{\circ}{a}} b_{j=1}{ }_{k j}{ }^{3} 0, \quad " k \\
& \stackrel{n}{\stackrel{n}{a}} 1_{r j}=1, \quad " j \\
& I_{r j}{ }^{3} 0, \quad " r, j .
\end{aligned}
$$

Considering the dual variables $v_{i}, u_{k}, u_{0 j}$ for all $\mathrm{i}, \mathrm{k}, \mathrm{j}$ corresponding to constraints of model (6), the dual model is as follows:

$$
\begin{aligned}
& \text { Maximize } \stackrel{n}{\stackrel{0}{a}} u_{0 j}
\end{aligned}
$$

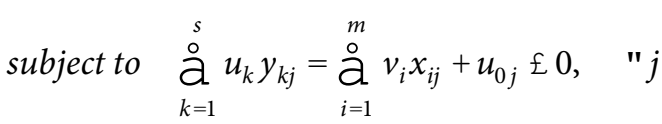

$$
\begin{aligned}
& v_{i} £ w_{i}, \quad " i \\
& u_{k} £ w_{k}, \quad " k \\
& v_{i}{ }^{3} 0, u_{k}{ }^{3} 0, \quad " i, k \text {. }
\end{aligned}
$$

By assigning values to parameters $w_{i}$ and $w_{k}^{k}$, model (5) is converted to model (6), which is a linear programming problem from whose optimal solution the gradient of the efficient hyperplanes can be determined.

\section{THEOREM 6}

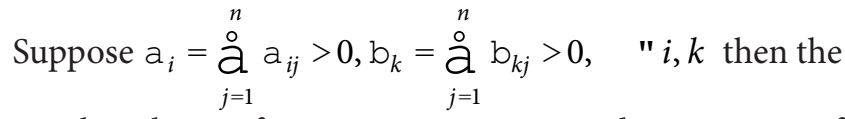
weights chosen from $w_{i}$ and $w_{k^{k}}$ are gradient vectors of the efficient hyperplane in $T_{v}$.

\section{ProOf:}

Suppose $\left(I_{r j}^{*}, a_{i}^{*}, b_{k}^{*}\right)$ is a Pareto optimal solution of

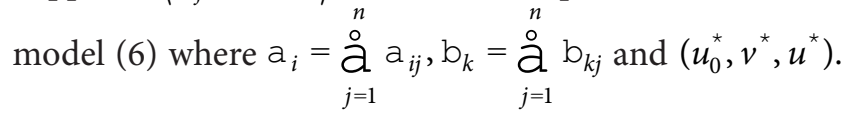


is a optimal solution of model (7). According to complementary slackness theorem $\mathrm{d}\left(w_{i}-v_{i}^{*}\right) \mathrm{a}_{i}^{*}=0, " i$, $\left(w_{k^{*}}-u_{k}^{*}\right) \mathrm{b}_{k}^{*}=0, " k$ and Because $\mathrm{a}_{i}^{*}>0, \mathrm{~b}_{k}^{\star}>0$ then $w_{i}=v_{i}^{\star}, w_{k^{k}}=u_{k}^{\star}$. Now by strong duality property $u_{0 j}^{\star}=\stackrel{\rho}{a}_{k=1}^{s} u_{k}^{\star} y_{k j}-\stackrel{\rho}{a}_{i=1}^{m} v_{i}^{\star} x_{i j}$, " $j$ because each pareto-optimal solution of model (5) is an efficient unit in $T_{v}$, so the weights that are chosen from $w_{i}$ and $w_{k}$ are the gradient of the efficient hyperplane in $T_{v}$.

\section{Z-W Method and Entropy Method}

Z-W method and Entropy method are used to solve the proposed model. When decision maker preferences are present, it is advisable to employ the $\mathrm{Z}-\mathrm{W}$ method, otherwise Entropy method is used.

\subsection{Z-W Method}

Many interactive methods have been developed for solving multi-objective linear programming during the years. In interactive methods, a solution pattern is formed and iteratively repeated, and the DM takes actively part in the solution process by specifying and refining preference information. In this way, the DM can learn about the possibilities and limitations of the problem and about the interdependencies among the objective functions. The method of Zionts-Wallenius (Z-W) can be used to design an interactive procedure for searching for most preferred solution (MPS) that maximizes the DM's implicit utility function. Zionts-Wallenius ${ }^{2}$ (Z-W) method was introduced by Zionts and Wallenius in 1976 and updated in 1983 .

It is applicable to problem where the objective functions are concave and is a convex set. Here, the objective

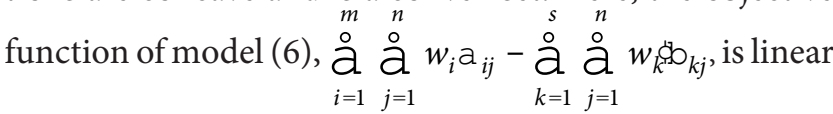
then it is concave and Limitations of the model (6) can be written as $A x=b, x \geq 0$. Then it is formed a convex set, so $\mathrm{Z}-\mathrm{W}$ method is used to solve the model (6). The method makes use of such an implicit function on an interactive basis. The first step of the method is to choose an arbitrary set of positive multipliers or weights, it is better to use

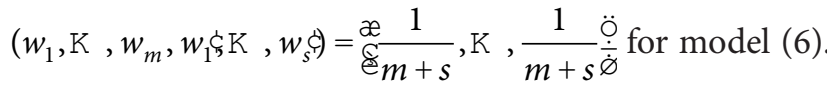
The composite objective function is then optimized and produced a solution that is an efficient unit to the problem because According to Theorem 1, the weights are positive so a Pareto optimal solution is obtained by solving the model (6) and Theorem 3 implies that each pareto optimal solution is a efficient unit. From the set of non basic variables, a subset of efficient variables is selected (an efficient variable is one which, when introduced into the basis, cannot increase one objective without decreasing at least one other objective). For each efficient variable a set of tradeoffs is defined by which some objectives are increased and others reduced. A number of such tradeoffs are presented to the $\mathrm{DM}$, who is requested to state whether the tradeoffs are desirable, undesirable or neither. From his/her answers a new set of consistent multipliers is constructed and the associated non dominated solution is found. The process is then repeated, and a new set of tradeoffs is presented to the DM at the current solution, convergence to an overall optimal solution with respect to the DMs implicit utility function is assured.

\subsection{Entropy Method}

The concept of Shannon's entropy ${ }^{23}$ has a central role in information theory, and sometimes refers to measure of uncertainty. This concept has been extended to different scientific fields, such as physics, social sciences, and so on. There are nine steps. First, we describe how if we have $\mathrm{n}$ DMUs, $\mathrm{s}$ output variables, and $\mathrm{m}$ input variables, we could perform the matrix as shown in (8). The columns represent the input variables $\left(X_{1}, X_{2}, \ldots, X_{m}\right)$, and the rows represent the decision making units $\left(D M U_{1}, D M U_{2}, \ldots\right.$, $\left.D M U_{n}\right)$

Step 1: From the input variables/DMU matrix

$$
\begin{aligned}
& X_{1} \mathrm{~K} \quad X_{j} \mathrm{~K} \quad X_{m} \\
& D M U_{1} \dot{e}_{\hat{e}} x_{11} L x_{1 j} L \quad x_{11} \text { ù } \\
& \text { M êM M M ú } \\
& D M U_{i} \hat{e}_{\hat{e}^{-}} x_{i 1} \mathrm{~L} x_{i j} \mathrm{~L} x_{i n} \text { ú } \\
& \text { M } \hat{e}_{\hat{e}} \mathrm{M} \quad \mathrm{M} \quad \mathrm{M} \text { ú } \\
& D M U_{n} \hat{e}^{x_{n 1}} \mathrm{~L} x_{n j} \text { L } x_{n m} \text { 兒 }
\end{aligned}
$$

Step 2: Find each cell $\left[P_{i j}\right]$ by dividing each element by the sum of all elements of $X_{i}$ :

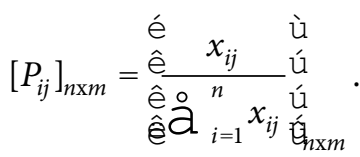


Step 3: Find the entropy for all variables.

$$
e_{j}=-k \stackrel{n}{\stackrel{\circ}{a}} P_{i j} \operatorname{Ln} P_{i j}
$$

where, $\mathrm{k}$ is Boltzman's constant, which is equal to

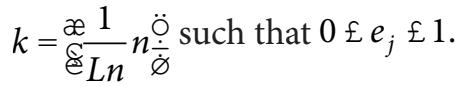

Step 4: Define the degree of diversification $\bar{e}_{j}$ of the information provided by the alternative DMU/variables and the value of variables $j$ as:

$$
\bar{e}_{j}=1-e_{j}
$$

Step 5: Normalize all variables

$$
r_{j}=\frac{\bar{e}_{j}}{{\stackrel{\circ}{a} \bar{e}_{j}}_{j=1}^{\circ},}
$$

where, $\stackrel{\circ}{a}_{j=1}^{n} r_{j}=1$. this allows us to find the weight of the input variables $\left[w_{1}, w_{2}, \ldots, w_{m}\right]$.

Step 6: Form the output variables/DMU matrix The columns represent the output variables $\left(Y_{1}, Y_{2}, \ldots, Y_{m}\right)$, and the rows represent the decision-making units $\left(D M U_{1}\right.$, $\left.D M U_{2}, \ldots, D M U_{n}\right)$ and repeat 2-5 to calculate the weight of the output variables $\mathrm{e}_{1}$

With these weights the objective function of model

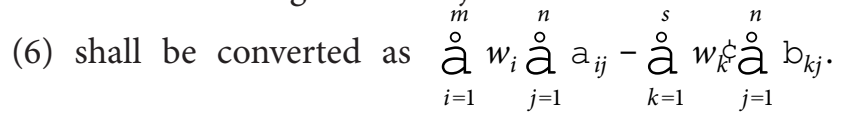
According to theorem 6, if $\mathrm{a}_{i}^{*}>0, \mathrm{~b}_{k}^{*}>0$ then the entropy weights are the gradient of the efficient hyperplane in $T_{v}$.

\section{A Numerical Example and an Application}

In this section, to illustrate the proposed models a numerical example and an application will be used. The numerical example is a single-input, single-output problem and the application is a three-input, three-output problem.

\subsection{A Numerical Example}

This example is a single-input, single-output problem. Table 1 shows the original DMUs as well as those to which they are projected using BCC-I and CRA and the proposed approach using entropy. Finally the example will be solved by Z-W method.

Note that the fourth column, the numerical example is solved using the entropy method and the entropy weights are $\left(w_{1}, w_{2}\right)=(1,1)$ whereby $\mathrm{D}$ is determined as the projection for all DMUs. Can see that D has the average value of the input and nearly the maximum output.

Now the numerical example is solved using $\mathrm{Z}-\mathrm{W}$ method. The general combined-oriented BCC model is run to find the respective efficiency scores, you can see, G, E, C are inefficient units. For example, consider G, its composite unit on the efficient frontier can be represented as a linear combination of 0.5 of $\mathrm{B}, 0.5$ of $\mathrm{D}$. In fact, the composite unit of $\mathrm{G}$ is given as follows: $(\mathrm{X}, \mathrm{Y})=$ $(4.5,9)$.This means the input (Payable interest) should be reduced from 8 to 4.5 and the output should be fixed. However, the DM is not accepted the DEA composite input and output values as the MPS for G. Hence, using interactive MOLP method is needed to search MPS along

\begin{tabular}{|c|c|c|c|c|c|c|c|c|}
\hline \multirow{2}{*}{ DMU } & \multicolumn{2}{|c|}{ Existing } & \multicolumn{2}{|c|}{ BCC-I } & \multicolumn{2}{|c|}{ Radial-CRA } & \multicolumn{2}{|c|}{$\begin{array}{l}\text { Proposed model } \\
\text { (using Entropy) }\end{array}$} \\
\hline & $\mathrm{x}$ & $\bar{y}$ & $x^{*}$ & $y^{*}$ & $\bar{x}$ & $\bar{y}$ & $\alpha$ & $\beta$ \\
\hline $\mathrm{A}$ & 3 & 3 & 3 & 3 & 4 & 8 & 5 & 10 \\
\hline B & 4 & 8 & 4 & 8 & 4 & 8 & 5 & 10 \\
\hline C & 5 & 5 & 3.4 & 5 & 3.6 & 6 & 5 & 10 \\
\hline $\mathrm{D}$ & 5 & 10 & 5 & 10 & 4 & 8 & 5 & 10 \\
\hline $\mathrm{E}$ & 6 & 8 & 4 & 8 & 4 & 8 & 5 & 10 \\
\hline $\mathrm{F}$ & 7 & 11 & 7 & 11 & 4 & 8 & 5 & 10 \\
\hline G & 8 & 9 & 9 & 4.5 & 4 & 8 & 5 & 10 \\
\hline
\end{tabular}
the frontier for $\mathrm{G}$.

Table 1. Numerical example data and results 
The first iteration of the interactive $\mathrm{Z}$-W method gives a unit as a linear combination of 0.85 of $\mathrm{F}$ and 0.34 of $\mathrm{D}$, as follows: $(\mathrm{X}, \mathrm{Y})=(6.43,10.71)$. The DM is still not satisfied with the solution obtained by first iteration.

In iteration 2, the solution is B. Now, the DM completely satisfied with the above input and output values. This means the MPS has been found and hence the interactive process terminate.

\subsection{Case Study: Taiwanese Commercial Bank Efficiency Evaluation}

To examine the capabilities of the entropy-based Russell measure (under constant returns to scale), we gathered information on 24 commercial banks in Taiwan to serve as a case study. Based on the applications of Miller and Noulas $^{21}$, the banking sector is regarded as an intermediary for bank transfers or deposits, even in the investment market. For this study, we used the amount of money deposited, employment expenses, and banking assets as input. Meanwhile, the amount of loans, commissions, and handling revenues and amount of investments are used as output. Table 1 shows the 24 commercial banks' input and output data sourced from the Taiwan Economics Journal (TEJ) database in 2007.

We used the entropy concepts in Section 4 to calculate the variable weights, as shown in Table 2. Table 3 shows the results of solving the model by the entropy method and CRA-BCC method. Finally, the application will be solved using Z-W method.

Table 2. Taiwanese Commercial Bank Raw Data

\begin{tabular}{|c|c|c|c|c|c|c|}
\hline \multirow[t]{2}{*}{ Bank } & \multicolumn{3}{|c|}{ Inputs } & \multicolumn{3}{|c|}{ Outputs } \\
\hline & Assets & Expense & Deposit & Commission & Loans & Investments \\
\hline 1 & 25,071 & 36,840 & $1,060,496$ & 2,977 & 875,145 & 264,084 \\
\hline 2 & 23,387 & 39,389 & $1,252,513$ & 5,569 & 979,891 & 360,010 \\
\hline 3 & 22,481 & 39,429 & $1,308,629$ & 4,823 & $1,030,504$ & 323,175 \\
\hline 4 & 1,360 & 2,270 & 19,531 & 361 & 73,903 & 153,166 \\
\hline 5 & 15,530 & 15,624 & $1,116,477$ & 6,095 & $1,126,008$ & 308,041 \\
\hline 6 & 3,892 & 6,405 & 171,819 & 345 & 142,982 & 4,772 \\
\hline 7 & 3,941 & 6,135 & 243,083 & 401 & 188,125 & 7,968 \\
\hline 8 & 34,902 & 42,237 & $1,153,181$ & 18,782 & 785,285 & 360,292 \\
\hline 9 & 23,823 & 32,260 & 911,310 & 5,694 & 674,607 & 383,584 \\
\hline 10 & 13,404 & 18,547 & 802,964 & 10,047 & 617,984 & 133,782 \\
\hline 11 & 14,767 & 19,137 & 883,675 & 1,637 & 756,745 & 143,393 \\
\hline 12 & 2,984 & 3,954 & 123,279 & 325 & 99,567 & 35,450 \\
\hline 13 & 7,966 & 9,855 & 209,363 & 1,453 & 140,005 & 18,432 \\
\hline 14 & 8,489 & 10,127 & 296,604 & 2,299 & 208,782 & 53,702 \\
\hline 15 & 10,971 & 7,492 & 766,250 & 2,112 & 595,122 & 218,067 \\
\hline 16 & 11,016 & 12,442 & 529,789 & 2,903 & 441,690 & 140,371 \\
\hline 17 & 3,088 & 4,407 & 281,145 & 1,346 & 229,770 & 31,939 \\
\hline 18 & 17,443 & 21,149 & 669,698 & 7,568 & 531,404 & 86,231 \\
\hline 19 & 2,471 & 4,063 & 266,267 & 2,245 & 225,261 & 29,054 \\
\hline 20 & 3,172 & 4,817 & 272,770 & 2,652 & 244,739 & 40,950 \\
\hline 21 & 3,879 & 3,454 & 238,495 & 1,003 & 232,504 & 30,388 \\
\hline 22 & 14,565 & 16,471 & 291,123 & 1,596 & 235,684 & 31,267 \\
\hline 23 & 5,428 & 6,539 & 213,888 & 1,411 & 181,613 & 27,557 \\
\hline 24 & 33,993 & 34,440 & $1,917,281$ & 2,889 & $1,734,526$ & 158,476 \\
\hline
\end{tabular}


Table 3. Efficiency and Results of CRA-BCC and Entropy method

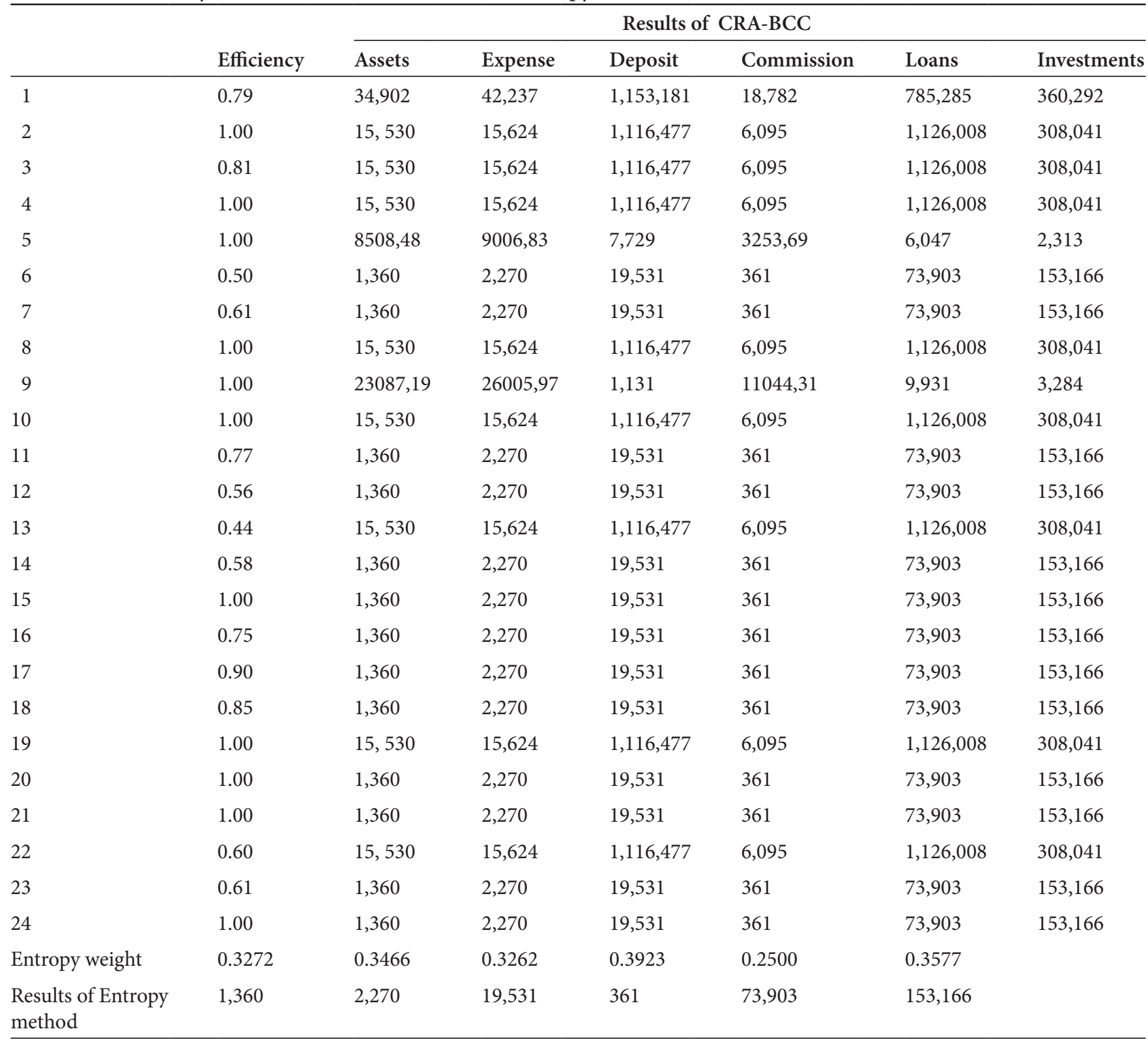

Bank 4 is determined as the projection for all DMUs. According to the data in Table 1, the first input of bank 4 is less than $30 \%$ of the first input of the other DMUs and the second input is less than $50 \%$ of the second input of the other DMUs and the third input is less than $20 \%$ of the first input of the other DMUs.

Now the application is solved using Z-W method. The general combined-oriented BCC model is run to find the respective efficiency scores, you can see 1, 3, 6, 7, 11, 12, $13,14,16,17,18,22,23$ are inefficient units. For example, bank 3 has an efficient score 0.81 implying that it is operating as an inefficient bank. We want to obtain its projection:

First the arbitrary weights are let $\left(w_{1}, \mathrm{~K}, w_{3}, w_{1}\right.$, $\left.\mathrm{K}, w_{3}^{\text {c }}\right)$ $={ }_{e}^{\infty} \frac{1}{6}, \mathrm{~K}, \frac{1}{6} \frac{\ddot{\dot{\phi}}}{\dot{\phi}}$ and model 7 is solved, efficient solution is given as follows;

$$
\begin{aligned}
& (\mathrm{I} 1, \mathrm{I} 2, \mathrm{I} 3)=(1360,2270,19531), \\
& (\mathrm{O} 1, \mathrm{O} 2, \mathrm{O} 3)=(361,73903,153166) .
\end{aligned}
$$

This means the first input (Assets) should be reduced from 22,481 to 1,360, the second input (Expense) should 
be reduced from 39,429 to 2,270 and the third input (Deposit) should be reduced from 1,308,629 to 19,531 for bank 3 to become efficient. Also, the Outputs O1 (Commission), O2 (Loans), O3 (Investments) should be reduce from 4,823 and $1,030,504$ and 323,175 to 361 and 73,903 and 153,166 respectively. However, the DM is not accepted solution as the MPS for bank 3. Hence, using interactive MOLP method is needed to search MPS along the frontier for bank 3 .

The first iteration of the interactive $\mathrm{Z}-\mathrm{W}$ method gives a unit as a linear combination of 0.01 of bank 2 and 0.47 of bank 8, 0.6 of bank 9 as follows:

$$
\begin{aligned}
& (\mathrm{I} 1, \mathrm{I} 2, \mathrm{I} 3)=(33993,34440,1917281), \\
& (\mathrm{O} 1, \mathrm{O} 2, \mathrm{O} 3)=(2889,1734526,158476) .
\end{aligned}
$$

The DM is still not satisfied with the solution obtained by first iteration. In iteration 2 , the solution is as a linear combination of 0.3 of bank 4 and 0.84 of bank 5 and 0.03 of bank 8 as follows:

$$
\begin{aligned}
& (\mathrm{I} 1, \mathrm{I} 2, \mathrm{I} 3)=(14500.26,15072.27,978295.41), \\
& (\mathrm{O} 1, \mathrm{O} 2, \mathrm{O} 3)=(5791.56,991576.17,315513) .
\end{aligned}
$$

Again, the DM is not accepted the solution of iteration 2 and carry on interactively to search for the MPS that satisfied all preferences. The third iteration of interactive $\mathrm{Z}-\mathrm{W}$ method gives a unit as follows:

$$
\begin{aligned}
& (\mathrm{I} 1, \mathrm{I} 2, \mathrm{I} 3)=(15530,15624,1116477), \\
& (\mathrm{O} 1, \mathrm{O} 2, \mathrm{O} 3)=(6095,1126008,308041) .
\end{aligned}
$$

Where it is bank 5 . Now, the DM completely satisfied with the above input and output values. This means the MPS has been found and hence the interactive process terminate.

\section{Conclusion}

In Data Envelopment Analysis (DEA), for each DMU a linear programming problem is solved and the efficiency score and the projection of the DMU are obtained. As interactive methods are not used, some solutions are ignored. As the entropy method obtains certain weights for input and output, using these weights in the proposed MOLP-CRA model is of paramount importance. Using the interactive $\mathrm{Z}-\mathrm{W}$ method for solving the proposed model, yields a projection corresponding to the viewpoint of the DM and the analyst, which is nearer to reality and more practical? This method obtains all pareto points. The assigned weights in the proposed weighted model are the gradient vector of the efficient hyperplane in $T_{v}$.
Our proposed MOLP-CRA model has three advantages over the basic DEA models: one being the decrease in the sum of inputs or the increase in the sum of outputs; another being the need for solving only one instead of $n$ linear programming models for obtaining the projection of all DMUs; and still a third advantage being the possibility for employing interactive methods, which yield a projection that is more realistic and practical. In the application provided in the previous section, $24 \mathrm{LP}$ problems must be solved for calculating the efficiency scores and determining the projection of the DMUs, whereas the projection of all DMUs can be obtained by solving our proposed only once. By using the interactive solution method, the most preferred projection can be determined.

When decision maker preferences are present, it is advisable to employ the Z-W method, whereby bank 5 is determined as the projection for all DMUs. Otherwise the entropy method is used and bank 4 is determined as the projection for all DMUs. As can be seen from the data in Table 1, the first input of bank 4 is less than $30 \%$ of the first input of the other DMUs and the second input is less than $50 \%$ of the second input of the other DMUs and the third input is less than $20 \%$ of the first input of the other DMUs.

In first, $\mathrm{Z}-\mathrm{W}$ method gives an efficient unit, in next iterations; according to DM it gives better solution. But the process has a lengthy computation. According to theorem 1, 5, the entropy weights are the gradient of the efficient hyperplane in $T_{v}$. Entropy Weights cannot be modified unless the inputs and outputs change. For future research, we suggest using the CRA model in the framework of the SBM and Russell models. Furthermore, determining return to scale by using the proposed model can be of great importance.

\section{References}

1. Athanassopoulos AD. Goal Programming and Data Envelopment Analysis (GODEA) models for multi-level multi-unit organizations: an application to Greek local authorities. Eur J Oper Res. 1995; 87(3):535-50.

2. Zionts S, Wallenius J. An interactive programming method for solving the multiple criteria problem. Manag Sci. 1976; 22:6563.

3. Yu MM, Chern, CC, Hasio B. Human resource rightsizing using centralized data envelopment analysis; evidence from Taiwan's airports. Omega. 2013; 41:119-30.

4. Yang J.-B, Wong BYH, Dong-Ling Xu, Stewart TJ. Integrating DEA-oriented performance assessment and 
target setting using interactive MOLP methods. Eur J Oper Res. 2009; 195:205-22.

5. Cooper WW. Origins, uses, and relations between goal programming and data envelopment analysis, MCDM 2004; Whistler BC, Canada. 2004. p. 6-11, 725-34.

6. Charnes A., Cooper WW, Rhodes E. Measuring the efficiency of decision making units. Eur J Oper Res. 1978; 2:429-44.

7. Doyle J, Green R. Data envelopment analysis and multiple criteria decision making. Omega. 1993; 21 (6):713-15.

8. Fang L. A generalized DEA model for centralized resource allocation. European Journal of Operation Research. 2013; 228:405-12.

9. Golany B. An interactive MOLP procedure for the extension of DEA to effectiveness analysis. J Oper Res Soc.1988; 39 (8).

10. Hadad Y, Friedman L, Rybalkin V, Sinuany-Stern Z. The relationship between DEA efficiency and the type of production function, the degree of homogeneity, and error variability. Cent Eur J Oper Res. 2013; 21:595-607

11. Hosseinzadeh Lotfi F, Jahanshahloo GR, Soltanifar M, Ebrahimnejad A, Mansourzadeh SM. Relationship between MOLP and DEA based on output-orientated CCR dual model. Expert Syst Appl. 2010; 37:4331-6.

12. Thanassoulis E, Dyson RG. Estimating preferred target input-output levels using data envelopment analysis. Eur J Oper Res. 1992; 56:80-97.

13. Hosseinzadeh Lotfi F. Noora AA, Jahanshahloo GR, Jablonsky J, Mozaffari MR, Gerami J. An MOLP based procedure for finding efficient units in DEA models. Cent Eur J Oper Res. 2008 Nov 15; 17(1):1-11.

14. Hsiao B, Chern $\mathrm{CH}$, Chiu $\mathrm{CH}$. Performance evaluation with the entropy-based weighted Russell measure in data envelopment analysis. Expert Syst Appl. 2011; 38(8):9965-72.

15. Hwang C, Masud A. Multiple Objective Decision Making Methods and Applications: A State-of-the-Art Survey, Springer, Berlin; 1979.

16. Jablonsky J. Multicriteria approaches for ranking of efficient units in DEA models, Cent Eur J Oper Res. 2012; 20(3):435-49.

17. Lozano S, Villa G. Centralized resource allocation using data envelopment analysis. J Prod Anal. 2004; 22:143-61.

18. Lozano S, Villa G, Adenso-Diaz B. Centralized target setting for regional recycling operations using DEA. OMEGA. 2004; 32:101-10.

19. Lozano S, Villa G. Multiobjective target setting in data envelopment analysis using AHP. Comput Oper Res. 2009; 36(2):549-64.

20. Steuer RE. Multiple Criteria Optimization: Theory, Computation, and Application. New York: Wiley. 1986.

21. Miller S. Noulas AG. The technical efficiency of larg bank production. J Bank Finance. 1996; 20:495-509.

22. Mar-Molinero C, Prior D, Segovia MM, Portillo F. Centralized resource utilization and its reallocation by using DEA. Ann Oper Res. 2012; 1-12.

23. Shannon CE. A mathematical theory of communication. Bell System Technical Journal. 1948; 27:379-423, 623-56. 